\title{
Identification of sub-grains and low angle boundaries beyond the angular resolution of EBSD maps
}

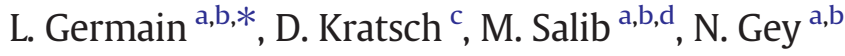 \\ a Laboratoire d'Etude des Microstructures et de Mécanique des Matériaux (LEM3), UMR 7239, CNRS/Université de Lorraine, F-57045 Metz, France \\ b Laboratory of Excellence on Design of Alloy Metals for low-mAss Structures ('LabEx DAMAS'), Université de Lorraine, France \\ c Laboratoire d'Informatique Théorique et Appliquée (LITA), EA3079, Université de Lorraine, 57045 Metz Cedex 1, France \\ d Institut Jean Lamour (IJL), SI2M Dept., CNRS UMR 7198, Université de Lorraine, Parc de Saurupt, CS 50840, F-54011 Nancy Cedex, France
}

\section{A R T I C L E I N F O}

\section{Keywords:}

EBSD

Sub-grain

low-angle boundaries

ALGrId

Grain Boundary Completion

Dijkstra algorithm

\begin{abstract}
A B S T R A C T
A new method called ALGrId (Anti-Leak GRain IDentification) is proposed for the detection of sub-grains beyond the relative angular resolution of Electron Backscatter Diffraction maps. It does not use any additional information such as Kikuchi Pattern Quality map nor need data filtering. It uses a modified Dijkstra algorithm which seeks the continuous set of boundaries having the highest average disorientation angle.
\end{abstract}

\section{Introduction}

The identification of sub-grains and low angle boundaries in EBSD (Electron Backscatter Diffraction) maps finds many applications in material sciences, for example, the detection of sub-grains in recovered or geometrically recrystallized materials [1], or the discrimination of closely oriented domains formed by variant selection after a phase transformation [2]. However, the detection of such boundaries in EBSD maps is limited by the relative angular resolution of the technique [3]. In this work, we propose a new method to detect sub-grains and low angle boundaries beyond the angular resolution of EBSD maps.

The relative angular resolution of an EBSD map is a function of multiple SEM and EBSD system parameters. A high angular resolution $\left(\sim 0.01^{\circ}\right)$ may be obtained using HR-EBSD (High Resolution-EBSD) [4]. With a conventional system and in optimal conditions, a disorientation angle can be measured with a precision lower than half a degree [5]. However, there is a tradeoff between angular resolution and the acquisition rate. If no particular care has been taken, the angular resolution is more reasonably of one degree [6,7].

The first section of this manuscript demonstrates that sub-grain detection using classical grain identification algorithms becomes unreliable

\footnotetext{
* Corresponding author at: Laboratoire d'Etude des Microstructures et de Mécanique des Matériaux (LEM3), UMR 7239, CNRS/Université de Lorraine, F-57045 Metz, France.

E-mail address: Lionel.germain@univ-lorraine.fr (L. Germain).
}

at low disorientation angles, especially when approaching the domain of orientation noise. Four methods have been published in the open literature on the detection of either low-angle boundaries or sub-grains on conventionally acquired EBSD maps. First, Humphreys et al. proposed to smooth the orientation noise using a modified Kuwahara filter [8]. Barou used the Kikuchi Pattern Quality (KPQ) map and the Band Slope ${ }^{1}$ as extra information to detect sub-grains [1,9]. McMahon et al. proposed using a clustering method [10]. Finally, Channel5 users may detect grains using the Grain Boundary Completion tool (GBC) [11]. However, all these methods except GBC use additional data or modify the initial data by filtering. The new method proposed in this paper was inspired by the GBC but works differently. It can be used to reconstruct sub-grains from raw orientation data (no filtering, no use of additional information such as KPQ).

The first part of this paper recalls the problems of grain detection at low disorientation angles. After, we describe our new method, called ALGrId (Anti-Leak GRain IDentification). Then, a validation test demonstrates that ALGrId detects sub-grains beyond the angular resolution. Finally, an application example illustrates the efficiency of the method to detect sub-grains in a deformed $\beta$ titanium alloy.

\footnotetext{
${ }^{1}$ The KPQ is also named Band Contrast. The Band Slope is an indicator solely available in Oxford instruments systems. It is an indicator of sharpness of band edges in Kikuchi patterns.
} 


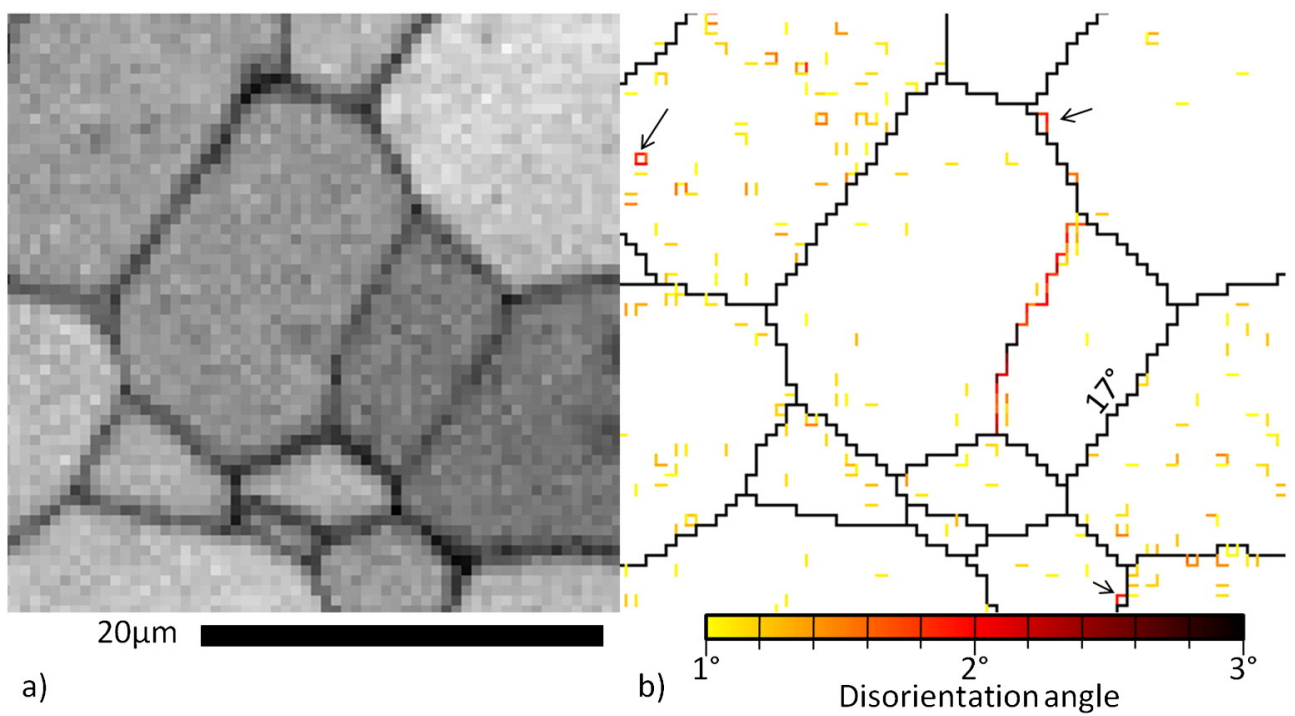

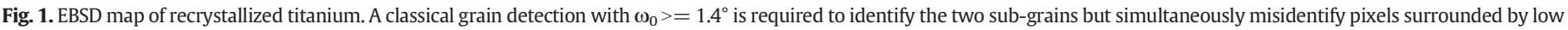
angle boundaries as sub-grains. a) KPQ map, b) disorientation angles map.

\section{Problems with the detection of sub-grains in EBSD maps}

There is a duality in the identification of sub-grains and low-angle boundaries that separate them. Grains can be identified from EBSD data as soon as they are delimited by a boundary having a disorientation angle above the relative angular resolution of the EBSD map [3]. Recommendations and good practices in this domain can be found in [12]. A "floodfill" algorithm is classically used to reconstruct grains in commercial software [13]. It requires a critical disorientation angle, $\omega_{0}$, set by the user. A grain is then defined as a cluster of connected pixels with neighboring pixel disorientation angles lower than $\omega_{0}$. The grain boundaries are the interfaces between those clusters and always have a disorientation angle greater than $\omega_{0}$. If the identification is correct, there is an agreement between grains and clusters.

Sub-grain boundaries may have a disorientation angle lower than the angular resolution of EBSD measurements and therefore, sub-grain identification using a floodfill algorithm generates artifacts: isolated pixels surrounded by boundaries with $\omega_{0}$ are misidentified as grains and separated sub-grains may be misidentified as a single grain. This is illustrated in Fig. 1. Pure titanium (T40) was scanned for EBSD acquisition in a Zeiss Supra 40 FEG SEM equipped with a Bruker E-Flash EBSD camera. Patterns were acquired with a speed of $\sim 100 \mathrm{~Hz}$ and a step size of $0.5 \mu \mathrm{m}$ was used. Classical cleaning was performed before analysis. ${ }^{2}$ This small map was extracted from a larger map containing more than 2500 grains. The central grain contains a low angle boundary with disorientation angles varying from $1.4^{\circ}$ to $3.2^{\circ}$. This boundary appears as clearly on the KPQ map as the $17^{\circ}$-boundary indicated in Fig. $1 \mathrm{~b}$. This confirms the sensitivity of the KPQ to low angle disorientation boundaries as reported in [9]. This sensitivity is used in the last section of this manuscript to validate the sub-grain detection on the application example. The grain in the top corner contains more "orientation noise" than other grains. As observed in [14], the angular resolution depends on the orientation of the grains. This example shows a typical case were no value of $\omega_{0}$ allows the discrimination of the two subgrains without misidentifying some isolated pixels as sub-grains (pointed by arrows in Fig. 1b). In the larger map out of which this data was taken, about $10 \%$ of the grains were "noisy" (i.e. in which small clusters were misidentified with $\omega_{0}=2^{\circ}$ ) and $1 \%$ of grains contained a low angle boundary with a misorientation angle greater than $2^{\circ}$.

\footnotetext{
${ }^{2}$ Grains detected with $\omega_{0}=10^{\circ}$ and smaller than 2 pixels were removed and a onestep dilatation of non-indexed pixels was performed.
}

Such an example is regularly encountered on EBSD maps and therefore we developed ALGrId to solve the problem.

\section{The Anti-Leak Grain Identification (ALGrId)}

We have developed a new method in order to identify sub-grains separated by boundaries with a disorientation angle lower than the relative angular resolution of the map. Specific vocabulary is required to understand the method (Fig. 2). In this work we need to make a distinction between a frontier (the separation between two pixels) and a boundary (a continuous set of frontiers). Each frontier is characterized by the disorientation between its two adjacent pixels. After grain identification, all grains are surrounded by boundaries with frontiers having a disorientation angle greater than $\omega_{0}$. These boundaries are called closed boundaries because they enclose the grains. Not all frontiers with a disorientation angle greater than $\omega_{0}$ belong to closed boundaries. In contrast, these are called open frontiers.

Our method aims to "close" the open frontiers. In other words, to find a boundary that connects an open frontier to a closed boundary (Fig. 2c). Potentially, many boundaries match this criterion. Among these, we define the optimum choice as having the maximum average disorientation angle (i.e. the sum of the disorientation angles of all frontiers contained in the boundary divided by the number of frontiers). However, it is impractical to iterate over all boundaries in order to find this optimum as this would run in a time proportional to an exponential of the number of pixels in the map. To solve the problem, we have implemented a modified Dijkstra algorithm [15].

The Dijkstra algorithm originally finds a path of minimum total weight in a weighted graph but it is here adapted to find our optimum boundary. A weighted graph is a representation of data in which nodes (or vertices) are connected with edges (or links). Each edge $E$ has a weight, $w(E) \in \mathbb{R}$. For more information on the graph theory and on the Dijkstra algorithm, one may read [16]. The Dijkstra algorithm finds a path (continuous set of edges) that goes from a node, $\mathrm{N}_{0}$, to all other nodes $\mathrm{N}_{\mathrm{i}}$ and that has the minimum total weight (sum of weights of the edges in the path).

An EBSD map of $\mathrm{X} \times \mathrm{Y}$ pixels may be described by a graph of $\mathrm{X} \times(\mathrm{Y}+$ $1)+1$ nodes and $2(X \times Y)+X+1$ edges. The data structure used is illustrated in Fig. 3 on a $4 \times 2$ pixels EBSD map. The pixels of the EBSD maps are named $\mathrm{P}_{\mathrm{i}}$. The frontiers are converted in edges. A distinction is made between horizontal and vertical edges marked VE and HE respectively. The numbering was chosen to avoid data conversion: each pixel $\mathrm{P}_{\mathrm{i}}$ is always surrounded by the nodes $\mathrm{N}_{\mathrm{i}}, \mathrm{N}_{\mathrm{i}+1}, \mathrm{~N}_{\mathrm{i}}+\mathrm{X}$ and $\mathrm{N}_{\mathrm{i}}+1+\mathrm{x}$ and by 

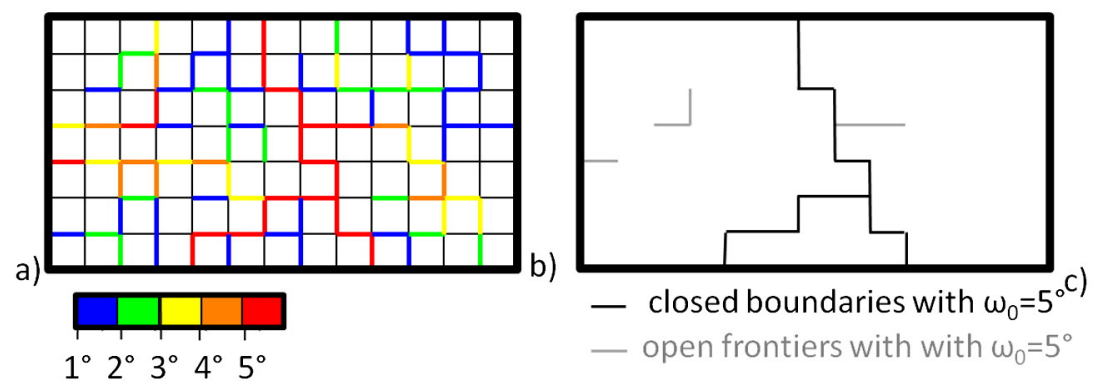

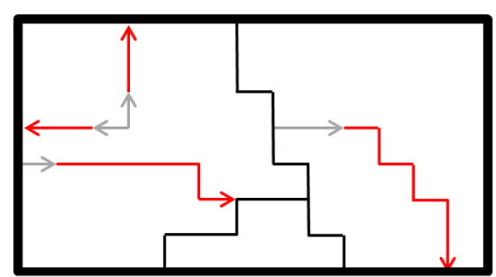

- New closed boundaries

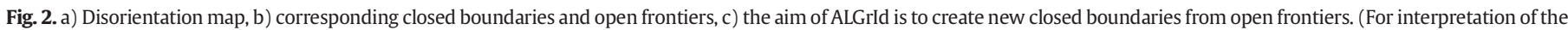
references to color in this figure legend, the reader is referred to the web version of this article.)

\begin{tabular}{|l|l|l|l|}
\hline$P_{0}$ & $P_{1}$ & $P_{2}$ & $P_{3}$ \\
\hline$P_{4}$ & $P_{5}$ & $P_{6}$ & $P_{7}$ \\
\hline
\end{tabular}

a)

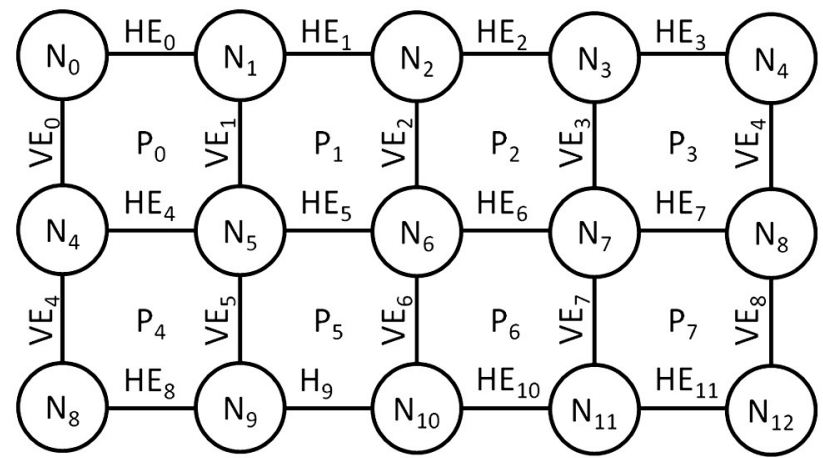

b)

Fig. 3. Conversion of EBSD data into a graph. Original EBSD map of $4 \times 2$ pixels (a) is converted into a graph (b).

edges $\mathrm{HE}_{\mathrm{i}}, \mathrm{HE}_{\mathrm{i}}+\mathrm{x}, \mathrm{VE}_{\mathrm{i}}, \mathrm{VE}_{\mathrm{i}}+1$. The drawback of this numbering is that some nodes and edge at the border of the map appear two times (see $\mathrm{N}_{4}, \mathrm{~N}_{8}$ and $\mathrm{VE}_{4}$ ). This does not interfere with the usage of the graph since the border of the map is considered as a potential end in the path search but allows significant time savings in data conversion. It would be also possible to use a sequential numbering of the node and edges but then no simple relation would exist between pixel, node and edge

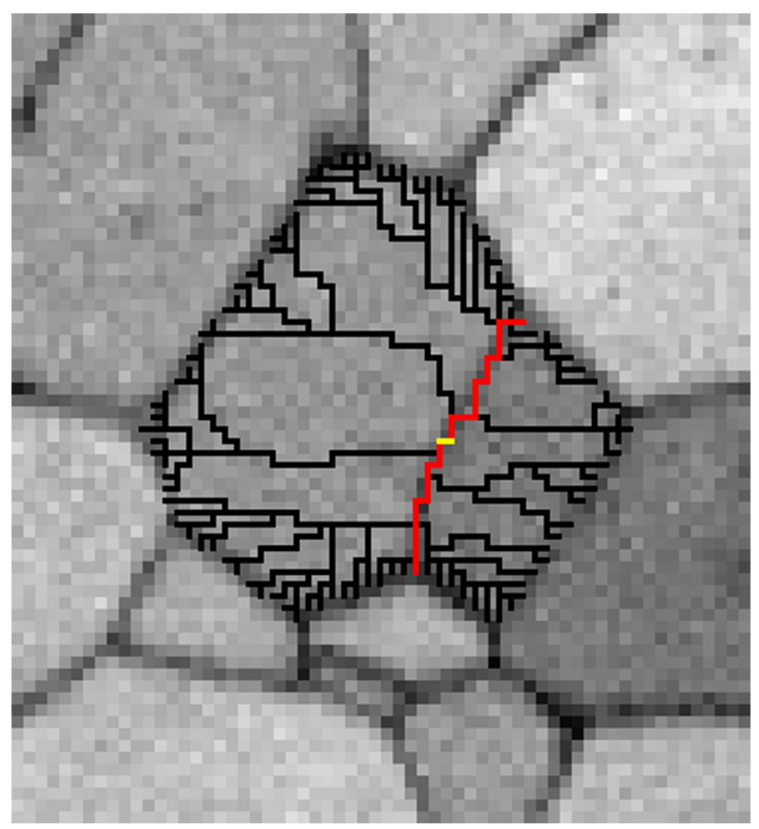

Fig. 4. Starting from the open frontier in yellow, all paths of minimum weight joining a closed boundary are browsed (in black). That of maximum average disorientation angle is the selected low angle boundary (in red). (For interpretation of the references to color in this figure legend, the reader is referred to the web version of this article.) numbering. Then to this purpose one would need to "build a specific graph".

To summarize, after conversion, each frontier becomes an edge of the graph and the search of a continuous set of frontiers becomes the search of a path in the graph.

The choice of the edge's weight is important. Any positive and decreasing function of the disorientation angle, $\omega$, gives satisfactory results but the following function was found to give the best results by trial and error:

$w(E)= \begin{cases}0, & \text { if } \omega \geq \omega_{0} \\ \frac{1}{\omega-\omega_{1}}-\frac{1}{\omega_{0}-\omega_{1}}, & \text { if } \omega_{1}<\omega<\omega_{0} . \\ \infty, & \text { if } \omega \leq \omega_{1}\end{cases}$

This function gives a very high weight to edges corresponding to low angle frontiers and a weight of zero to open frontiers. Consequently, the Dijkstra algorithm prioritizes the boundaries with the highest disorientation angle because it attempts to minimize the sum of the path. The weighting function also prevents closing boundaries with frontiers with a disorientation angle lower than $\omega_{1}$ (set by the user). A choice of $\omega_{1}=0$ is possible (not necessarily recommended) and would result in the closing of all open frontiers in the EBSD map. In the Dijkstra algorithm, the origin is a node. In our problem, the origin has to be an open frontier (i.e. an edge), so the two nodes connected to that edge are taken simultaneously as the origin. The original algorithm stores information at each node and updates it at each calculation step ${ }^{3}$. In our modified Dijkstra, at each node we need to store and update two additional pieces of information: the origin node and the length of the path.

\footnotetext{
3 This information is the predecessor of a node and the total weight.
} 


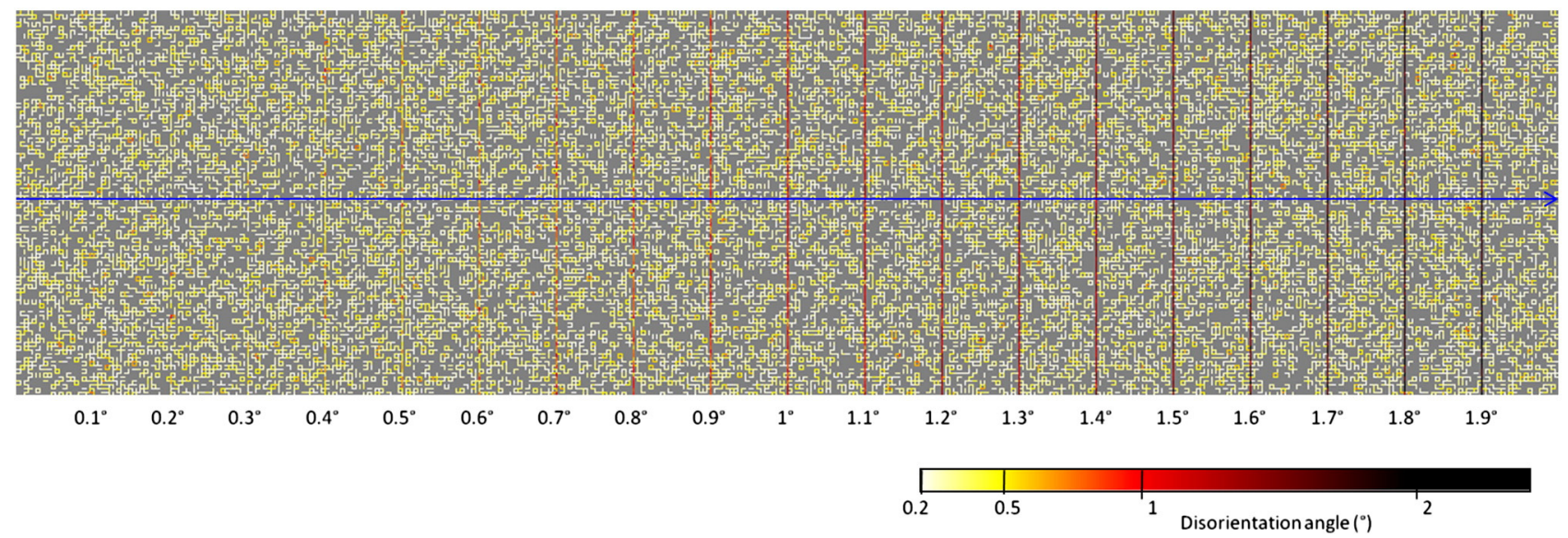

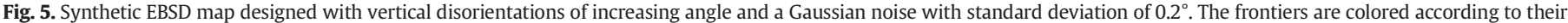
disorientation angle. The background is in gray. (For interpretation of the references to color in this figure legend, the reader is referred to the web version of this article.)

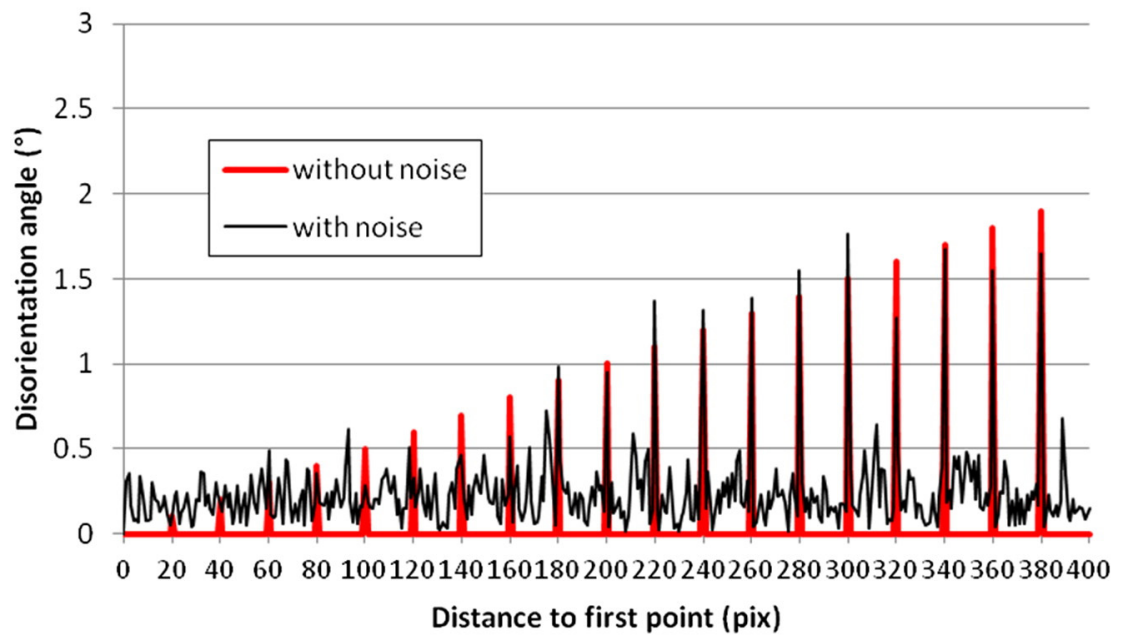

Fig. 6. Pixel to pixel disorientation profile along the blue line (Fig. 5) compared with the disorientation without the noise.

The result of a run $\left(\omega_{0}=3^{\circ}, \omega_{1}=0^{\circ}\right)$ on the central grain of Fig. 1 is presented in Fig. 4. The open frontier used as the origin is indicated in yellow. A minimum weight path joining the origin to any node of the surrounding closed boundary has been found and is displayed in black. There are as many paths as there are pixels on the perimeter of the grain. All frontiers are not contained in any one path. Instead, the paths favor frontiers of maximum disorientation angle because of the weight function. The picture would be different with $w(E)=1$. Then, the paths would be on the radii of a circle centered on the origin.

For each path, the average weight is calculated. It is the total weight divided by the length of the path. From both nodes taken as origin, the path with the lowest average weight is kept as a solution. It correspond also to the boundary with the highest average disorientation angle. In Fig. 4 the two paths are plotted in red. They correspond to the detected low-angle boundary and split the grain into two sub-grains.

Other adaptations are required to take into account the specificities of EBSD data:

- The user chooses if the border of the EBSD map is treated like a closed boundary or not (in other words, if closing is possible at the border of the map or not).

- The best way to handle non-indexed pixels is to start with a grain detection using the MTEX routine [17] which has the advantage of detecting grains without cleaning. Then, all edges adjacent to nonindexed pixels are allocated a weight with $\omega$ set at the angular resolution of the EBSD map. Alternatively, another approach is to consider edges adjacent to non-indexed pixels like closed boundaries. The only exceptions are edges adjacent to non-indexed pixels that are totally included in a grain which are allocated a weight with $\omega$ set at the angular resolution of the EBSD map. This allows boundary closing through non-indexed points rather than having to find a path around them.

- If a grain contains several sub-grains, the choice of starting frontier (processing sequence) may slightly influence the results. Therefore, it is preferable to process the open frontiers in decreasing order of

Table 1

Summary of the most interesting results obtained on the synthetic EBSD map of Fig. 5.

\begin{tabular}{|c|c|c|c|c|}
\hline & $\begin{array}{l}\omega_{0} \\
\left({ }^{\circ}\right)\end{array}$ & $\begin{array}{l}\omega_{1} \\
\left({ }^{\circ}\right)\end{array}$ & $\begin{array}{l}\text { Lowest disorientation } \\
\text { angle boundary } \\
\text { identified } \\
\left({ }^{\circ}\right)\end{array}$ & Artifacts \\
\hline ALGrId & 0.9 & 0.2 & $0.4^{\circ}$ & None \\
\hline $\begin{array}{l}\text { Classical Grain } \\
\text { identification }\end{array}$ & 0.8 & - & $1.3^{\circ}$ & None \\
\hline $\begin{array}{l}\text { Classical Grain } \\
\text { identification }\end{array}$ & 0.7 & - & $1.1^{\circ}$ & 8 isolated pixels \\
\hline GBC & 1.4 & $\geq 0.2$ & $0.9^{\circ}$ & None \\
\hline GBC & 1 & $0.7^{\circ}$ & 0.7 & 12 isolated pixels \\
\hline
\end{tabular}




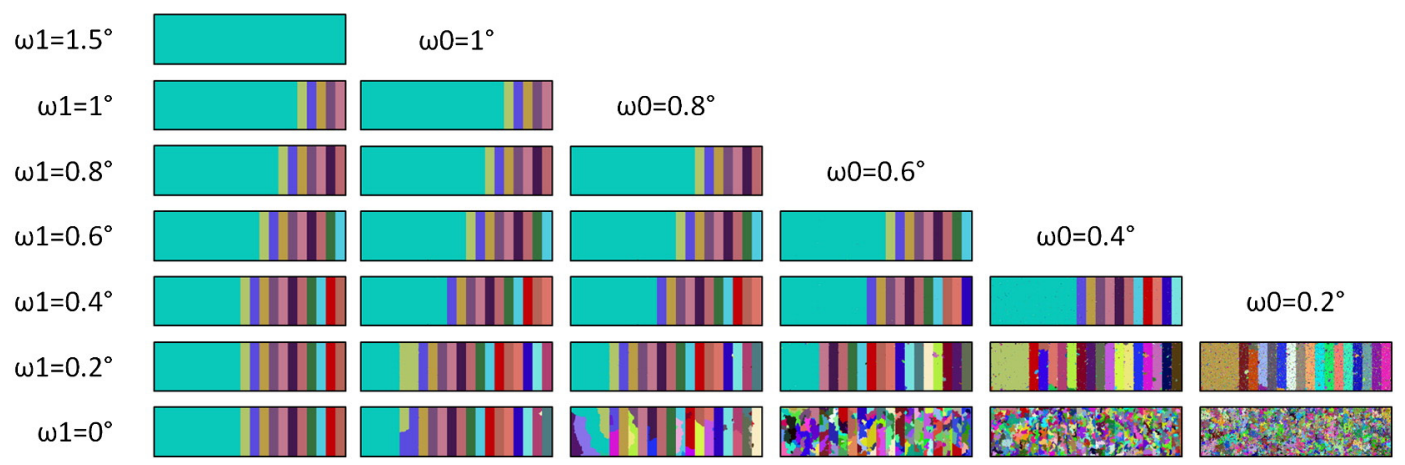

Fig. 7. Some results using ALGrId with different parameters to detect grains in the map of Fig. 5 (Grains in random colors). (For interpretation of the references to color in this figure legend, the reader is referred to the web version of this article.)
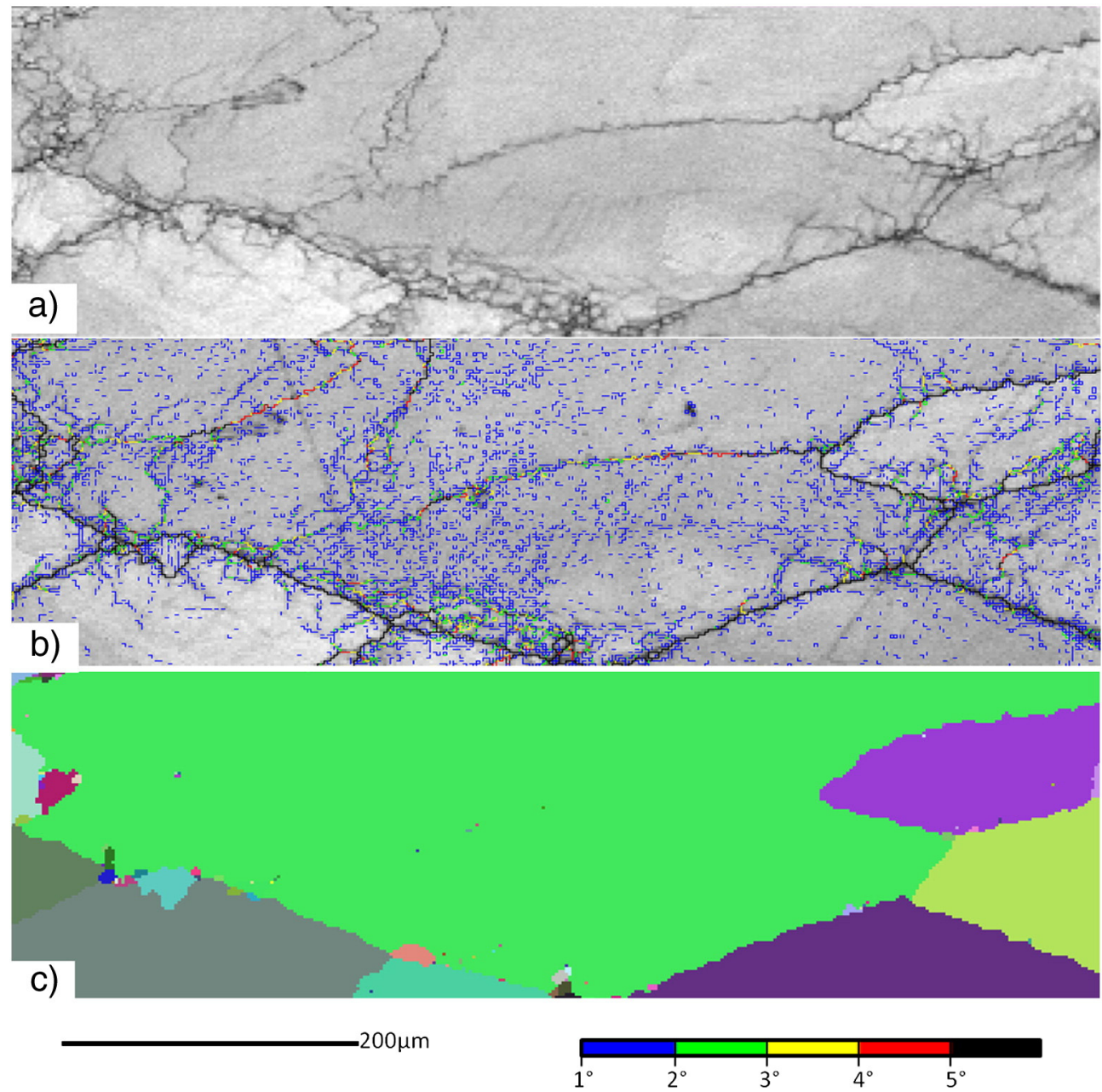

Fig. 8. Deformed Ti 17 titanium alloy EBSD map, a) KPQ map, b) disorientation map, c) classical grain identification with $\omega_{0}=2$.

disorientation angle. Once a low-angle boundary is detected, it is considered equal to other closed boundaries in the next iteration.

\section{Validation of the method}

ALGrId was tested on a synthetic EBSD map $(400 \times 100$ pixels $)$ containing 19 regularly-spaced vertical boundaries with a disorientation angle varying from $0.1^{\circ}$ to $1.9^{\circ}$ with a step of $0.1^{\circ}$. A Gaussian orientation noise was added. Each pixel was rotated around a random axis by a random angle. The random angles were chosen to follow a centered Gaussian distribution with a standard deviation of $0.2^{\circ}$. The resulting map is shown in Fig. 5.

The pixel to pixel disorientation profile along the blue line in Fig. 5 is shown in Fig. 6 . In between the authentic boundaries, an average noise of $0.2^{\circ}$ with some peaks at $0.7^{\circ}$ was measured. In the whole map, the maximum orientation noise reached $1^{\circ}$. In Fig. 6 , the first boundary that is clearly distinguishable from the noise has a disorientation angle of $0.9^{\circ}$ ( situated at 180 pixels). 


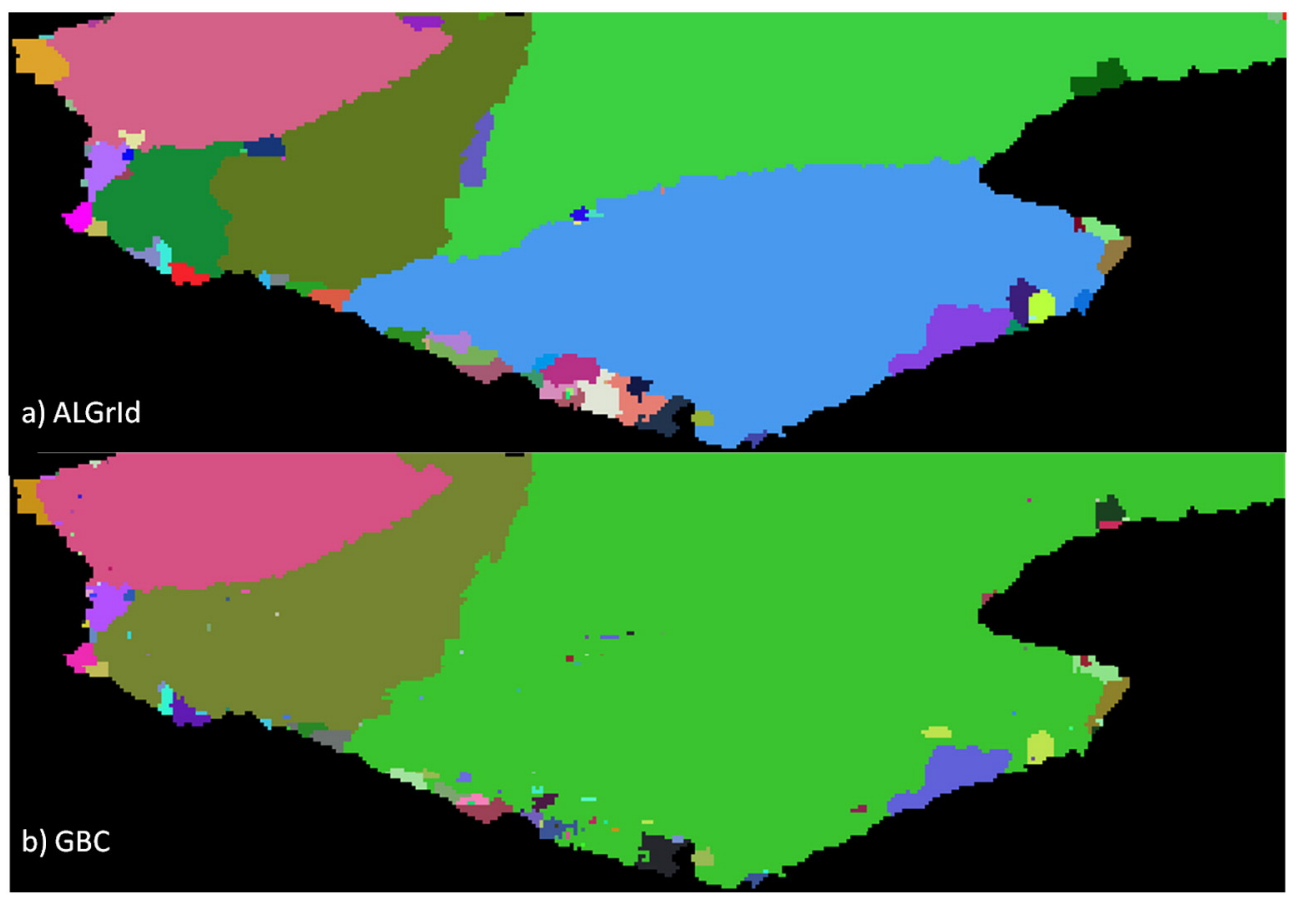

Fig. 9. Detection of sub-grains in the central grain of Fig. 8. Each sub-grain is colored randomly a) the result of ALGrId, b) the result of GBC.

ALGrId was applied to the synthetic map with $\omega_{0}$ and $\omega_{1}$ ranging from $2^{\circ}$ down to $0^{\circ}$ with a step of $0.1^{\circ}$. The most relevant results are reported in Table 1 and others are sampled in Fig. 7.

Let us focus first on results where $\omega_{0}=\omega_{1}$ which are equivalent to the classical grain identification (Fig. 7). Boundaries with lower disorientation angles are detected with decreasing values of $\omega_{0}$. Artifacts are generated when $\omega_{0} \leq 0.7$ ( 8 isolated pixels are misidentified in the case with $\omega_{0}=\omega_{1}=0.7^{\circ}$ ). In this case, the lowest disorientation angle boundary detected is $1.1^{\circ}$. Considerable improvement is seen using ALGrId. The best run is obtained with $\omega_{0}=0.9^{\circ}$ and $\omega_{1}=0.2^{\circ}$ and successfully detects the $0.4^{\circ}$ disorientation angle boundary with no artifacts. Any other set of parameters doesn't improve the resolution but creates artifacts (see Fig. 7). The results of the GBC (presented in Table 1 but not in Fig. 7) are in between ALGrId and the classical grain detection.

ALGrId gives the best results with $\omega_{0}$ being slightly above the peak disorientation angle of the orientation noise and $\omega_{1}$ set at the average noise level. If no easy evaluation of the noise level is possible a compromise can be found by taking $\omega_{0}$ sufficiently high to ensure that the all open frontiers really belong to a boundary. Then any choice of $\omega_{1}$ in the noise level should provide reliable results but not the best achievable resolution (see for example the first column in Fig. 7).

\section{Application to sub-grain detection in $\beta$-deformed Ti-17 Titanium alloy}

A Ti-17 titanium alloy was $\beta$-deformed by tension $(\varepsilon=32 \%)$ at a temperature of $920^{\circ} \mathrm{C}$ and then helium quenched. An FEI QUANTA 600 FEG microscope equipped with an Oxford Instruments EBSD system (Nordlys F camera) was used to acquire the map shown in Fig. 8 (the step size was $2 \mu \mathrm{m}$ ). A typical $\beta$-deformed substructure was obtained $[18,19]$. Serrated $\beta$-grain boundaries with well developed sub-grains are clearly seen on the KPQ map (Fig. 8a). The corresponding subgrain boundaries (Fig. 8b) mostly have disorientation angles lower than $3^{\circ}$ (only few boundary segments are delimited by $5^{\circ}$ disorientations). The classical grain reconstruction with $\omega_{0}=2^{\circ}$ fails to detect the sub-grains and creates artifacts (Fig. 8c)
Both the GBC and ALGrId methods were applied to the central grain (with parameters $\omega_{0}=3^{\circ}$ and $\omega_{1}=0.3^{\circ}$ ). The resulting grain detections are given in Fig. 9.

Both methods show some improvement with respect to classical grain identification. ALGrId detected successfully the sub-grains that are visible on the KPQ image (Compare Figs. 8a and 9a). On the contrary, the GBC detected only some sub-grains accurately (Fig. 9b). However, it missed some obvious boundaries and simultaneously created some artifacts. In all cases, ALGrId gave better results than GBC. Any comparison of both methods should also include a comparison of simplicity and rapidity. In this respect, the GBC runs faster. Our implementation of the Dijkstra algorithm is optimized for fast running speed (use of a binary heap [16]). This allows ALGrId to run in a reasonable time. ${ }^{4}$

\section{Conclusions}

The classical method to identify grains fails when applied to boundaries having disorientation angles of the same order as the relative angular resolution of EBSD. In this case, artifacts are generated.

A new method to detect sub-grains and/or low angle boundaries beyond the angular resolution of EBSD is proposed. It is called ALGrId (Anti-Leak Grain Identification) and finds in an EBSD map boundaries of high average disorientation angle. This new method improves the detection of grains compared to the classical algorithm and to Grain Boundary Completion.

Applied to deformed titanium, it successfully identified sub-grains without creating artifacts.

\section{Acknowledgements}

The authors acknowledge Prof. F. Wagner (LEM3) for providing the T40 EBSD map as well as K. Larsen from Oxford Instruments for the description of the GBC algorithm. The authors really appreciated the implication of both reviewers whose comments and questions did not

\footnotetext{
${ }^{4}$ Approximately proportional to $\mathrm{N} \log (\mathrm{N})$, that is a grain detection takes usually 23 min on a $10^{6}$ pixels EBSD map.
} 
only improve the quality of the paper but also lead to ideas for the improvement of ALGrId's algorithm.

\section{References}

[1] F. Barou, C. Maurice, J.-M. Feppon, J. Driver, Sub-grain boundary mobilities during recovery of binary Al-Mn alloys, Int. J. Mater. Res. 100 (2009) 516-521, http://dx. doi.org/10.3139/146.110057.

[2] L. Germain, N. Gey, M. Humbert, Reliability of reconstructed [beta]-orientation maps in titanium alloys, Ultramicroscopy 107 (2007) 1129-1135, http://dx.doi.org/10. 1016/j.ultramic.2007.01.012.

[3] F.J. Humphreys, Review grain and subgrain characterisation by electron backscatter diffraction, J. Mater. Sci. 36 (2001) 3833-3854, http://dx.doi.org/10.1023/A: 1017973432592.

[4] C. Maurice, R. Quey, R. Fortunier, J.H. Driver, High angular resolution EBSD and its materials applications, in: D.A. Molodov (Ed.), Microstruct. Des. Adv. Eng. Mater, Wiley-VCH Verlag GmbH \& Co. KGaA, 2013, pp. 339-365.

[5] F.J. Humphreys, Y. Huang, I. Brough, C. Harris, Electron backscatter diffraction of grain and subgrain structures - resolution considerations, J. Microsc. 195 (1999) 212-216, http://dx.doi.org/10.1046/j.1365-2818.1999.00579.x.

[6] N.C. Krieger Lassen, The relative precision of crystal orientations measured from electron backscattering patterns, J. Microsc. 181 (1996) 72-81, http://dx.doi.org/ 10.1046/j.1365-2818.1996.95376.X.

[7] V. Randle, Microtexture determination and its applications, Maney for the Institute of Materials, Minerals and Mining2003.

[8] F.J. Humphreys, P.S. Bate, P.J. Hurley, Orientation averaging of electron backscattered diffraction data, J. Microsc. 201 (2001) 50-58, http://dx.doi.org/10.1046/j.13652818.2001.00777.x.
[9] F. Barou, (PHD Thesis) École Nationale Supérieure des Mines de Saint-Étienne, 2009.

[10] C. McMahon, B. Soe, A. Loeb, A. Vemulkar, M. Ferry, L. Bassman, Boundary identification in EBSD data with a generalization of fast multiscale clustering, Ultramicroscopy 133 (2013) 16-25, http://dx.doi.org/10.1016/j.ultramic.2013.04.009.

[11] Disclosed anonymously, Grain boundary completion, Res. Discl. (2013) (Www. researchdisclosure.com).

[12] International Organization for Standardization. ISO 13067:2011 - Microbeam analysis - electron backscatter diffraction - measurement of average grain size n.d.

[13] Flood fill. Wikipedia Free Encycl 2014.

[14] D.J. Prior, Problems in determining the misorientation axes, for small angular misorientations, using electron backscatter diffraction in the SEM, J. Microsc. 195 (1999) 217-225, http://dx.doi.org/10.1046/j.1365-2818.1999.00572.x.

[15] E.W. Dijkstra, A note on two problems in connexion with graphs, Numer. Math. 1 (1959) 269-271, http://dx.doi.org/10.1007/BF01386390.

[16] T.H. Cormen, C.E. Leiserson, R.L. Rivest, C. Stein, Introduction to Algorithms, 3rd ed The MIT Press, Cambridge, Mass, 2009.

[17] F. Bachmann, R. Hielscher, H. Schaeben, Grain detection from 2d and 3d EBSD data specification of the MTEX algorithm, Ultramicroscopy 111 (2011) 1720-1733, http://dx.doi.org/10.1016/j.ultramic.2011.08.002.

[18] N. Allain-Bonasso, F. Wagner, S. Berbenni, D.P. Field, A study of the heterogeneity of plastic deformation in IF steel by EBSD, Mater. Sci. Eng. A 548 (2012) 56-63, http:// dx.doi.org/10.1016/j.msea.2012.03.068.

[19] D.L. Bourell, H.J. McQueen, Thermomechanical processing of iron, titanium, and zirconium alloys in the bcc structure, J. Mater. Shap. Technol. 5 (1987) 53-73, http:// dx.doi.org/10.1007/BF02833686. 